\title{
Systemic air embolism as a complication of CT-guided percutaneous core needle lung biopsy: A case report and review of the literature
}

\author{
CHUANSHU SUN ${ }^{1}$, JIE BIAN ${ }^{1}$, SHENGYUAN LAI ${ }^{1}$ and XIUHUA LI ${ }^{2}$ \\ Departments of ${ }^{1}$ Radiology and ${ }^{2}$ Oncology, The Second Affiliated Hospital of Dalian Medical University, \\ Dalian, Liaoning 116027, P.R. China
}

Received August 26, 2014; Accepted June 22, 2015

DOI: $10.3892 / \mathrm{etm} .2015 .2643$

\begin{abstract}
Computed tomography (CT)-guided percutaneous core needle biopsy (PCNB) of the lung is a widely accepted and frequently performed interventional radiological procedure for the diagnosis of various pulmonary lesions. Common complications are usually mild and self-limited; however, air embolism is an extremely rare but potentially fatal complication. This study reports a case of fatal air embolism in the coronary and spinal arteries that resulted from a complex CT-guided PCNB of the lung. The present case suggests that multiple precautions may not eliminate the risk of the fatal air embolism resulting from the procedure of CT-guided PCNB. Prompt recognition and urgent resuscitation are crucial for initial stabilization, allowing subsequent diagnostic confirmation and appropriate treatment. The common characteristics of symptomatic air embolism, based on the case reported and a review of the literature, are summarized in the present study in order to provide recommendations for clinical practice.
\end{abstract}

\section{Introduction}

Computed tomography (CT)-guided percutaneous core needle biopsy (PCNB) of the lung is a widely accepted and frequently used interventional radiological procedure that aims to diagnose pulmonary pathological conditions. Common complications include pneumothorax and pulmonary hemorrhage, both of which are known to be generally self-resolving with no clinical intervention required. Air embolism is a potentially life-threatening but extremely rare complication, with an estimated incidence of $0.02-0.06 \%(1,2)$; however, the incidence of air embolism is considered to be underestimated

Correspondence to: Dr Xiuhua Li, Department of Oncology, The Second Affiliated Hospital of Dalian Medical University, 467 Zhongshan Road, Dalian, Liaoning 116027, P.R. China E-mail: drlixh@163.com

Key words: air embolism, computed tomography, image-guided lung biopsy due to undiagnosed asymptomatic patients (3-5). The present study reports a case of air embolism in the coronary and spinal arteries, which, following a CT-guided PCNB of the lung, resulted in sudden mortality. In addition, a summary of the most common characteristics of symptomatic air embolism, based on the present case and a review of the literature, is presented in this study.

\section{Case report}

A 53-year-old woman visited the Second Affiliated Hospital of Dalian Medical University (Dalian, China), with a 15-day history of a productive cough. No indications of previous abnormalities were identified in the patient's history. Chest CT-scan results showed multiple nodular and flake-shaped lesions in both lungs. It was recommended that the patient be referred for a CT-guided PCNB of the lung for pulmonary histopathological diagnosis. Prior to the procedure, the patient was fully informed about all the details of the procedure and subsequently signed a written consent form. The following examinations were performed: Blood pressure, electrocardiogram, blood cell count and coagulation function tests, including activated partial thromboplastin and prothrombin time tests. All results were found to be within the normal ranges.

The CT-guided PCNB procedure was performed by a radiologist with 11 years of experience with PCNB. A 17-gauge introducer needle and an 18-gauge cutting biopsy needle (length, $13 \mathrm{~cm}$; TSK Laboratory, Tochigi, Japan) were selected for the procedure. During the procedure, the patient was placed in a prone position and instructed to hold her breath when required. Prior to biopsy, a CT scan of the thorax was performed using a Siemens Somaton Volume Zoom CT scanner (Siemens, Inc., Erlangen, Germany) with an 8-mm slice thickness and 8-mm intervals to determine the flake-shaped lesion in the left lower lobe adjacent to the chest wall as the target of biopsy.

Once the patient had been moved into the CT gantry and placed in the desired position, the needle entry site was marked with indelible ink and radiopaque locators using a gantry laser alignment light. A brief CT scan was repeated at a position $2 \mathrm{~cm}$ above and $2 \mathrm{~cm}$ below the selected slice during suspended respiration. Based on these images, the appropriate table position and needle trajectories were selected. 
The biopsy procedure was conducted as follows: Following the administration of local anesthesia via an injection of $2 \%$ lidocaine, a 17-gauge introducer needle was inserted into the prepped skin until the tip of the needle had reached the parietal pleura. Under CT guidance, the introducer needle was then advanced with a single motion along the planned trajectory to the prescribed depth required to reach the surface of the lesion. A CT scan was subsequently obtained to confirm the position of the needle tip (Fig. 1). Once the needle tip position had been confirmed, the internal stylet was removed and immediately replaced by the biopsy needle, which was advanced into the lesion for specimen procurement. Once the sample was obtained, the biopsy needle was removed and immediately replaced by the stylet of the introducer needle. Specimen acquisition was repeated twice and three core biopsy samples were obtained in total; the samples were then fixed in $10 \%$ neutral buffered formalin. Finally, the introducer needle was removed. During the procedure, the patient remained immobile on the CT table, did not cough and held her breath following moderate expiration, as instructed.

Following the withdrawal of the needle, the patient exhibited mild cough and hemoptysis. A postprocedural CT scan of the entire thorax was performed, and a small pneumothorax was observed (Fig. 2). Following the removal of the patient from the gantry, the radiologist noted that the patient was unresponsive and the pulse of the carotid artery was not palpable. Although immediate cardiopulmonary resuscitation (CPR) was performed with $100 \%$ oxygen $\left(\mathrm{O}_{2}\right)$ administration through a respirator, the patient could not be revived and succumbed on the CT table $\sim 1 \mathrm{~h}$ after the biopsy. A review of the postprocedural CT images revealed a considerable volume of air in the descending aorta and left ventricle and a smaller volume in the coronary and spinal arteries (Figs. 2 and 3). These findings led to the diagnosis of air embolism of the left ventricle and systemic arterial circulation. Histopathology of the obtained specimen revealed inflammatory lesions.

\section{Discussion}

CT-guided PCNB is a well-established and minimally invasive procedure for the diagnosis of indeterminate pulmonary lesions. Cases of air embolism as a complication of CT-guided PCNB are extremely rare, but also life-threatening $(1,2)$; however, the incidence rate of air embolism is believed to have been underestimated due to undiagnosed asymptomatic cases. Single-institutional, retrospective, observational studies based on the evaluation of CT scan images have suggested a higher incidence (3-5). Hiraki et al (3) found four cases of air embolism among 1,010 CT-guided lung biopsies based on postprocedural CT scans of the entire thorax, which gave an incidence rate of $0.4 \%$. Ibukuro et al (4) estimated an incidence rate of $0.21 \%$, based on a retrospective review of 1,400 CT-guided lung biopsies. Of note, Freund et al (5) showed that the radiological incidence of systemic air embolism complicating PCNB was 3.8\%, whereas the clinically apparent incidence was $0.49 \%$. The finding that the radiological incidence rate of air embolism is considerably higher than the clinically apparent incidence rate indicates that asymptomatic cases can lead to an overall underdiagnosis of this complication. Despite this higher radiological incidence rate, however, air embolism remains a relatively rare occurrence.
The present study described a case of fatal air embolism in the coronary and spinal arteries, which occurred immediately after a CT-guided PCNB of the lung. The patient was the first case of systemic air embolism among 1,100 lung biopsies performed over an 11-year time span, since CT-guided PCNB was introduced at the Second Affiliated Hospital of Dalian Medical University. To the best of our knowledge, this is also the first case of CT scan-confirmed air embolism in the spinal artery.

Despite the uncommonness of air embolism, its consequences can potentially be fatal; the occurrence of systemic air embolism can cause a rapid deterioration of the patient's cardiac and/or neurological condition, and requires prompt management and specialized treatment (5). The clinical manifestations of air embolism vary, depending on the exact location of the arterial embolus and the volume of air disseminated into the vessels. Once air enters the systemic circulation, it is distributed to respective arterial end beds. The systems most vulnerable to end-arterial occlusion by these bubbles are the cerebral and coronary circulation systems, which are sometimes affected simultaneously $(3,5)$. Even if only a small volume of air enters the systemic arterial circulation, functional end arteries can be occluded by air bubbles. An experimental study has demonstrated that $2 \mathrm{ml}$ air directly injected into the cerebral circulation is sufficient to have a fatal effect, and that $0.5-1.0 \mathrm{ml}$ air injected into the coronary artery can lead to cardiac arrest (6). The patient of the present study suffered sudden cardiac arrest and immediate loss of consciousness following the lung biopsy. According to the postprocedural chest CT-scan images, we propose that cardiac arrest as a result of coronary air embolism directly led to neurological lesions, due to potential spinal artery embolism, and then sudden mortality; however, the possibility of air embolisms in the cerebral circulation cannot be excluded without confirmation by a brain CT scan. Despite promptly providing CPR with $100 \%$ $\mathrm{O}_{2}$ administration, the severity of the condition did not allow further diagnosis or definitive therapy.

Due to the frequent performance of CT-guided PCNB of the lung, case reports of air embolisms complicating the procedure have been increasing in number. The characteristics of symptomatic air embolism, based on the present and previously reported cases, are summarized in the following section (3,7-24). In the majority of cases, the clinical symptoms of air embolism occur either during or immediately following needle biopsy. Shi et al (23), however, reported a case with a delayed onset ( $6 \mathrm{~h}$ after the procedure). Subjects with symptomatic air embolism usually present with a rapid deterioration of cardiac and/or cerebral status, which can result in sudden mortality. The occurrence of air embolism appears to have no association with the size of the biopsy needle (17-gauge to 23-gauge) or the biopsy methods, such as fine needle aspiration, needle core biopsy, automated core biopsy and coaxial needle biopsy. Air embolism has occurred when patients have suspended respiration during eupnea and at the end of inhalation or expiration. In these symptomatic cases, the lesions that underwent biopsy ranged from peripheral to hilar lesions, and the pulmonary parenchyma was penetrated at a needle depth of 0.5-6.3 cm (3). Pulmonary lesions included diffuse, ground-glass opacities, cystic lesions, solid masses and benign and malignant tumors. 


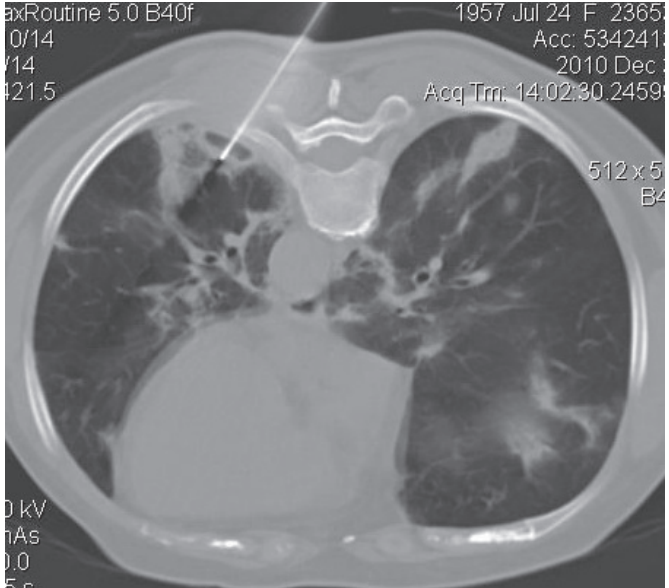

Figure 1. Computed tomography image obtained during biopsy showing multiple diffuse flake shadows in both lungs. The biopsy needle was introduced into the lesion in the posterior segment of the left inferior lobe after penetrating the pulmonary parenchyma at a depth of $0.5 \mathrm{~cm}$.

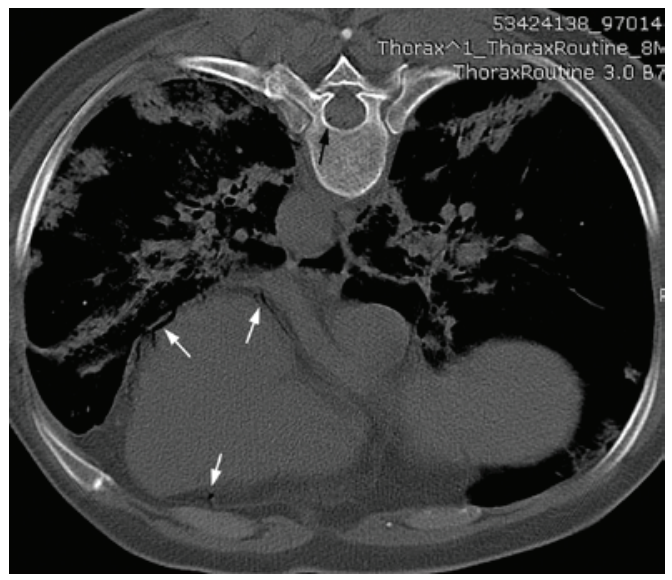

Figure 2. Postprocedural computed tomography image showing air in the coronary arteries (white arrows) and spinal canal (black arrow). A small pneumothorax is shown in the left lung.

The mechanism of air embolism complicating needle lung biopsy remains unclear, even following autopsy (20); however, a number of hypotheses have been proposed regarding possible pathways whereby air is introduced into the systemic circulation during a needle biopsy of the lung $(9,10)$. First, air may directly enter the pulmonary vein through the needle when the tip of the biopsy needle is lodged in a pulmonary vein and communicates between the pulmonary vein and the atmosphere when the inner stylet is removed and the atmospheric pressure exceeds the pulmonary venous pressure. Secondly, when a needle simultaneously traverses the alveolus and the pulmonary artery, air can access the pulmonary arterial system and reach the pulmonary venous circulation by traversing the pulmonary microvasculature. Thirdly, bronchial-venous or alveolar-venous fistulas may develop when a needle simultaneously traverses an air-containing space (e.g., alveolar, bronchus, cavitary or cystic lesion) and adjacent pulmonary vein, allowing air to enter the pulmonary vein when the patient coughs or the Valsalva maneuver is performed, both of which can increase airway pressure beyond pulmonary venous pressure. Cases of air embolism without any obvious triggers (e.g. cough, Valsalva maneuver and positive

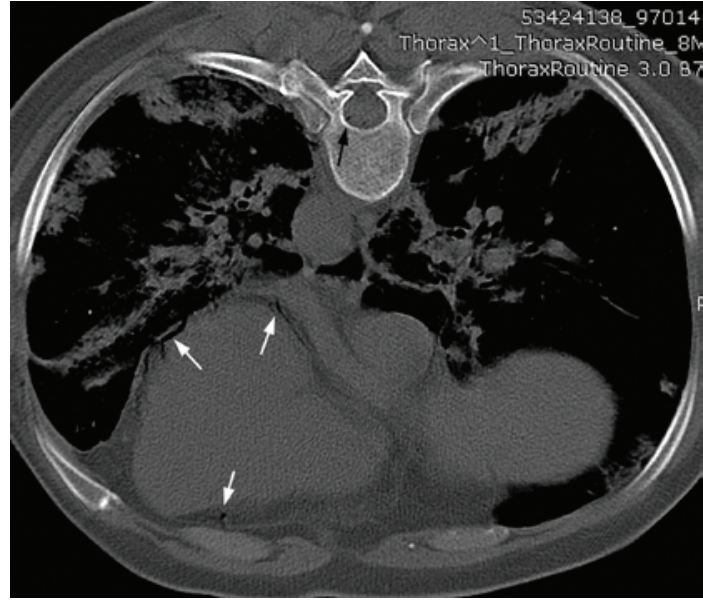

Figure 3. Postprocedural computed tomography image showing a large volume of air in the descending aorta (white arrow) and some in the spinal artery (black arrow).

pressure ventilation) may be associated with a quiet expiration that forwards air into the pulmonary vein. In the present study, it appeared improbable that air had entered the pulmonary vein directly through the needle, since the hollow of the needle was kept occluded at all times and the patient suspended respiration as instructed during the biopsy. Considering the rapid deterioration of the patient's condition and the large volume of air detected in the systemic circulation by CT scan, we hypothesized that the formation of an alveolar-venous or bronchial-venous fistula was the most probable cause of air embolism.

Studies have indicated that pathological pulmonary abnormalities may predispose a patient to an increased risk of air embolism $(12,24)$. It is believed that, following lung biopsy and needle withdrawal, a large number of alveolar-venous or bronchial-venous fistulas can develop along the needle trajectory. During vascular injury, the walls of small vessels usually retract, adhere spontaneously and become sealed. Extravasated and then coagulated blood may promote extravascular tamponade, which can prevent air in the alveoli or bronchi from entering the vein; however, pathological pulmonary abnormalities, such as inflammation, forms of vasculitis, e.g. Wegener's granulomatosis, and interstitial disease, which are risk factors of air embolism, may interfere with the healing process and result in prolonged exposure of the vessel lumen to the airway $(3,24)$.

Ghafoori and Varedi (11) presumed that an increased probability of air embolism could be associated with the size of the needle and the coaxial techniques. It has been suggested that larger gauge needles are associated with an increased risk of involving the pulmonary vein and coaxial system, which, following the removal of the internal stylet, is associated with an increased risk of contact with the atmosphere. This postulation, however, is controversial, since a case of systemic air embolism that used fine needles and did not involve the coaxial method has been reported (21).

Identifying the risk factors for air embolism and understanding their mechanisms will facilitate the development and implementation of adequate preventive measures prior to and during biopsy. Several precautionary measures have been proposed in order to minimize the risk of air embolism, such as avoiding biopsy through cystic or cavitary lesions or bullae, using a stylet to keep 
the needle closed airtight throughout the procedure, instructing the patient to suspend breathing during manipulation of the biopsy needle and minimizing the amount of aerated lung tissue penetrated while attempting to reach the lesion $(12,22)$.

In the present case, the clinical suspicion of systemic air embolism was based on the initial clinical manifestation: A rapid deterioration of the neurological/cardiovascular status. The postprocedural CT scan of the entire thorax, rather than just the target area of the biopsy, was essential in order to provide a definitive diagnosis and to detect evidence of air density in the pulmonary vein, left atrium and ventricle and systemic circulation (7). We propose that a CT scan of the brain be considered whenever manifestations of cerebral abnormalities occur during or following lung biopsy.

When a patient exhibits any suspicious symptoms of air embolism during biopsy, the needle has to be instantly removed. In such a case, it is recommended that the patient is placed in either the right lateral decubitus or Trendelenburg position, which can prevent air from entering the systemic circulation from the left ventricle, with immediate administration of $100 \% \mathrm{O}_{2}(25)$. Prompt CPR is necessary for a patient with a potentially fatal air embolism, and the stabilization of vital signs is the primary goal of treatment. In addition, hyperbaric $\mathrm{O}_{2}$ therapy is a definitive therapy for systemic air embolism and has been demonstrated to improve survival rate and neurological recovery. This therapy should be administered as soon as initial stabilization has been achieved $(8,12)$.

In the present case, a fatal air embolism complicating CT-guided lung biopsy occurred, despite the proximity of the lesion that underwent biopsy to the chest wall. During the procedure, the patient did not cough and was instructed to suspend breathing when required. The biopsy was performed by an experienced radiologist. Although previous studies have made certain recommendations and proposed certain precautions that could contribute to the reduction of the risk of air embolism, none of these proposals has succeeded in completely removing the risks. In order to achieve optimal outcomes for the patient, prompt recognition and urgent resuscitation are crucial for initial stabilization, following which a diagnostic confirmation can be made and definite treatments can be initiated. Standardized protocols for the early recognition and management of this rare but life-threatening complication should be developed.

\section{Acknowledgements}

The authors would like to thank Professor Changhong Liu and Professor Xu Zhang of the Department of Thoracic Surgery and Professor Ning Zhu of the Department of Cardiology of the Second Affiliated Hospital, Dalian Medical University for their contributions to this study.

\section{References}

1. Richardson CM, Pointon KS, Manhire AR and Macfarlane JT: Percutaneous lung biopsies: A survey of UK practice based on 5,444 biopsies. Br J Radiol 75: 731-735, 2002.

2. Tomiyama N, Yasuhara Y, Nakajima Y, Adachi S, Arai Y, Kusumoto M, Eguchi K, Kuriyama K, Sakai F, Noguchi M, et al: CT-guided needle biopsy of lung lesions: A survey of severe complication based on 9783 biopsies in Japan. Eur J Radiol 59: 60-64, 2006
3. Hiraki T, Fujiwara H, Sakurai J, Iguchi T, Gobara H, Tajiri N, Mimura $\mathrm{H}$ and Kanazawa S: Nonfatal systemic air embolism complicating percutaneous CT-guided transthoracic needle biopsy: Four cases from a single institution. Chest 132: 684-690, 2007.

4. Ibukuro K, Tanaka R, Takeguchi T, Fukuda H, Abe S and Tobe K: Air embolism and needle track implantation complicating CT-guided percutaneous thoracic biopsy: Single-institution experience. AJR Am J Roentgenol 193: W430-W436, 2009.

5. Freund MC, Petersen J, Goder KC, Bunse T, Wiedermann F and Glodny B: Systemic air embolism during percutaneous core needle biopsy of the lung: Frequency and risk factors. BMC Pulm Med 12: 2, 2012.

6. Ho AM and Ling E: Systemic air embolism after lung trauma. Anesthesiology 90: 564-575, 1999.

7. Ohashi S, Endoh H, Honda T, Komura N and Satoh K: Cerebral air embolism complicating percutaneous thin-needle biopsy of the lung: Complete neurological recovery after hyperbaric oxygen therapy. J Anesth 15: 233-236, 2001.

8. Sato K, Miyauchi K, Shikata F, Murakami T, Yoshioka S and Kawachi K: Arterial air embolism during percutaneous pulmonary marking under computed tomography guidance. Jpn J Thorac Cardiovasc Surg 53: 404-406, 2005.

9. Mansour A, AbdelRaouf S, Qandeel M and Swaidan M: Acute coronary artery air embolism following CT-guided lung biopsy. Cardiovasc Intervent Radiol 28: 131-134, 2005.

10. Cheng HM, Chiang KH, Chang PY, Chou YF, Huang HW, Chou AS and Yen PS: Coronary artery air embolism: A potentially fatal complication of CT-guided percutaneous lung biopsy. Br J Radiol 83: e83-e85, 2010.

11. Ghafoori M and Varedi P: Systemic air embolism after percutaneous transthoracic needle biopsy of the lung. Emerg Radiol 15: 353-356, 2008.

12. Um SJ, Lee SK, Yang DK, Son C, Kim KN, Lee KN and Kim YS: Four cases of a cerebral air embolism complicating a percutaneous transthoracic needle biopsy. Korean J Radiol 10: 81-84, 2009.

13. Bou-Assaly W, Pernicano P and Hoeffner E: Systemic air embolism after transthoracic lung biopsy: A case report and review of literature. World J Radiol 2: 193-196, 2010.

14. Higashino T, Noma S, Nishimoto Y, Endo J, Taguchi Y and Shindo T: Cerebral air embolism as a complication of computed tomography-guided marking of the lung: Depiction of air inflow route from a pulmonary vein to the left atrium. J Thorac Imaging 26: W26-W29, 2011.

15. Hirasawa S, Hirasawa H, Taketomi-Takahashi A, Morita $\mathrm{H}$, Tsushima Y, Amanuma M and Endo K: Air embolism detected during computed tomography fluoroscopically guided transthoracic needle biopsy. Cardiovasc Intervent Radiol 31: 219-221, 2008.

16. Lederer W, Schlimp CJ, Glodny B and Wiedermann FJ: Air embolism during CT-guided transthoracic needle biopsy. BMJ Case Rep 30: 2011, 2011.

17. Wu YF, Huang TW, Kao CC and Lee SC: Air embolism complicating computed tomography-guided core needle biopsy of the lung. Interact Cardiovasc Thorac Surg 14: 771-772, 2012.

18. Singh A, Ramanakumar A and Hannan J: Simultaneous left ventricular and cerebral artery air embolism after computed tomographic-guided transthoracic needle biopsy of the lung. Tex Heart Inst J 38: 424-426, 2011.

19. Kuo HL, Cheng L and Chung TJ: Systemic air embolism detected during percutaneous transthoracic needle biopsy: Report of two cases and a proposal for a routine postprocedure computed tomography scan of the aorto-cardiac region. Clin Imaging 34: 53-56, 2010.

20. Arnold BW and Zwiebel WJ: Percutaneous transthoracic needle biopsy complicated by air embolism. AJR Am J Roentgenol 178: 1400-1402, 2002.

21. Kamiyoshihara M, Sakata K, Ishikawa S and Morishita Y: Cerebral arterial air embolism following CT-guided lung needle marking. Report of a case. J Cardiovasc Surg (Torino) 42: 699-700, 2001.

22. Lattin G Jr, O'Brien W Sr, McCrary B, Kearney P and Gover D: Massive systemic air embolism treated with hyperbaric oxygen therapy following CT-guided transthoracic needle biopsy of a pulmonary nodule. J Vasc Interv Radiol 17: 1355-1358, 2006.

23. Shi L, Zhang R, Wang Z and Zhou P: Delayed cerebral air embolism complicating percutaneous needle biopsy of the lung. Am J Med Sci 345: 501-503, 2013.

24. Kau T, Rabitsch E, Celedin S, Habernig SM, Weber JR and Hausegger KA: When coughing can cause stroke-a case-based update on cerebral air embolism complicating biopsy of the lung. Cardiovasc Intervent Radiol 31: 848-853, 2008.

25. Balsara ZN and Burks DD: Hyperbaric oxygen therapy for arterial air embolism. AJR Am J Roentgenol 188: W98, 2007. 